\title{
Co-registration of laser altimeter tracks with digital terrain models and applications in planetary science
}

\author{
P. Gläser ${ }^{\text {a,*, I. Haase }}{ }^{\text {a }}$, J. Oberst ${ }^{\mathrm{a}, \mathrm{b}}$, G.A. Neumann ${ }^{\mathrm{c}}$ \\ a Technical University Berlin, Department of Geodesy and Geoinformation Science, Str. des 17. Juni 135, 10623 Berlin, Germany \\ ${ }^{\mathrm{b}}$ German Aerospace Center, Institute of Planetary Research, Rutherfordstrasse 2, 12489 Berlin, Germany \\ ${ }^{\mathrm{c}}$ NASA Goddard Space Flight Center, Code 698, Greenbelt, MD 20771, USA
}

\section{A R T I C L E I N F O}

\section{Article history:}

Received 25 April 2013

Received in revised form

28 August 2013

Accepted 16 September 2013

Available online 25 September 2013

\section{Keywords:}

Co-registration

LOLA

Laser

DTM

LROC

\begin{abstract}
A B S T R A C T
We have derived algorithms and techniques to precisely co-register laser altimeter profiles with gridded Digital Terrain Models (DTMs), typically derived from stereo images. The algorithm consists of an initial grid search followed by a least-squares matching and yields the translation parameters at sub-pixel level needed to align the DTM and the laser profiles in 3D space. This software tool was primarily developed and tested for co-registration of laser profiles from the Lunar Orbiter Laser Altimeter (LOLA) with DTMs derived from the Lunar Reconnaissance Orbiter (LRO) Narrow Angle Camera (NAC) stereo images. Data sets can be co-registered with positional accuracy between $0.13 \mathrm{~m}$ and several meters depending on the pixel resolution and amount of laser shots, where rough surfaces typically result in more accurate co-registrations. Residual heights of the data sets are as small as $0.18 \mathrm{~m}$. The software can be used to identify instrument misalignment, orbit errors, pointing jitter, or problems associated with reference frames being used. Also, assessments of DTM effective resolutions can be obtained. From the correct position between the two data sets, comparisons of surface morphology and roughness can be made at laser footprint- or DTM pixel-level. The precise co-registration allows us to carry out joint analysis of the data sets and ultimately to derive merged high-quality data products. Examples of matching other planetary data sets, like LOLA with LRO Wide Angle Camera (WAC) DTMs or Mars Orbiter Laser Altimeter (MOLA) with stereo models from the High Resolution Stereo Camera (HRSC) as well as Mercury Laser Altimeter (MLA) with Mercury Dual Imaging System (MDIS) are shown to demonstrate the broad science applications of the software tool.
\end{abstract}

(c) 2013 Elsevier Ltd. All rights reserved.

\section{Introduction}

Images and topographic data are fundamental for the exploration of planetary surfaces. Topography on planetary missions is typically derived by laser altimetry in the form of profiles acquired along the spacecraft ground track (Smith et al., 2000, 2011; Smith and Zuber, 2000). Alternatively, photogrammetric reduction of stereo images is used to produce gridded Digital Terrain Models (DTMs) (Preusker et al., 2012; Scholten et al., 2012; Oberst et al., 2010b; Haase et al., 2012). The laser profiles benefit from highly accurate individual point measurements, while the DTMs benefit from a contiguous large-area coverage. While both data sets are typically in good qualitative agreement, detailed comparisons often reveal lateral and vertical offsets in the raw data sets, in particular, when approaching the resolution limits of DTM pixel or laser spot sizes. In this work we have refined the method to precisely co-register and determine offsets between the two data

\footnotetext{
* Corresponding author. Tel.: +493031428828.

E-mail address: philipp.glaeser@tu-berlin.de (P. Gläser).
}

sets (Gläser et al., 2010). These offsets offer valuable insights into various instrumental or operational effects, but may also be of great scientific interest. Also, the availability of precisely coregistered data sets offers great opportunities of joint analysis to improve accuracy, detail, and coverage of final data products.

\section{Previous work}

The Mars Global Surveyor (MGS) imaged the Martian surface with the Mars Orbiter Camera (MOC) and the Mars Orbiter Laser Altimeter (MOLA). Kim et al. (2000) used visible edges of surface features such as craters to co-register MOLA tracks with MOC and VIKING orbiter images. The proposed approach, however, can only find horizontal shifts between the two data sets if a crater can be detected in each of them. The accuracies of the shift also highly depend on the hierarchical and heuristic edge detection method that is used and are not mentioned in this work. Yoon and Shan (2005) showed a combined adjustment of MOC stereo images and MOLA data. Trajectory data, tie points of the images, MOLA ranges, and MOLA surface points are adjusted simultaneously in this work 
using least-squares techniques. While an improvement for the ground location of MOLA surface points can be achieved, no condition is introduced in the algorithm minimizing the displacement between the stereo DTM and the MOLA profiles. Kolb and Okubo (2009) introduced a program to help co-registering MOLA data with Mars images obtained by MOC, the High Resolution Imaging Science Experiment (HiRISE) and the Context Camera (CTX). The user has to apply lateral shifts between images and MOLA tracks, but cannot align the two data sets in the 3-D space. No information on the fit accuracies can be retrieved in this approach. Di et al. (2012) and Lin et al. (2010) amongst others use surface matching techniques to register laser derived DTMs with stereo DTMs. Di et al. (2012) show surface matching using Chang'E-1 stereo images and crossover corrected laser DTMs (LAM) whereas Lin et al. (2010) match MOLA DTMs with High Resolution Stereo Camera (HRSC) and HiRISE DTMs. In both approaches the laser DTMs will contain tracks suffering from positional errors and interpolated data gaps.

\section{Data sets}

Several data sets were used to demonstrate the broad science applications of the software tool.

\subsection{LOLA}

The Lunar Reconnaissance Orbiter (LRO), launched in 2009, is equipped with the laser altimeter LOLA (Lunar Orbiting Laser Altimeter) and the camera system LROC (LRO Camera) featuring a pair of narrow- and one wide-angle camera (briefly termed NAC Narrow Angle Camera and WAC - Wide Angle Camera). LOLA generates single laser pulses transmitted through a diffractive optical element, which splits up the laser pulse into five individual beams. For each beam the time of flight (range), pulse spreading (surface roughness) and the ratio of transmitted/returned energy (surface reflectance) are measured. From the pattern of the five laser spots, surface slopes not only along but also across the orbiter's track can be determined. From LRO's nominal orbit altitude of $\sim 50 \mathrm{~km}$, LOLA covers a $\sim 50 \mathrm{~m}$ wide swath and the LOLA laser spot sizes are $5 \mathrm{~m}$ in diameter. At the laser pulse rate of $28 \mathrm{~Hz}$, along- and across-track spacing of the spots is $10-12 \mathrm{~m}$. The nominal ranging precision of the instrument is $10 \mathrm{~cm}$ and its vertical precision is $<1 \mathrm{~m}$ (Smith et al., 2011). In this work we used parts of a LOLA track from orbit 3508 (DOY 090 2010, primary mission phase).

\subsection{LROC NAC}

The two NACs, NAC-L and NAC-R, as part of the LROC system, are line scanners (5000 pix/line), operated in push-broom mode. From the nominal orbit height of $50 \mathrm{~km}$, the two NAC cameras cover a $5 \mathrm{~km}$ swath with a length of $\sim 25 \mathrm{~km}$ at an image scale of $0.5 \mathrm{~m} /$ pix. For the production of DTMs, multiple observations from different orbits and appropriate convergence angles are required. Typically, one nadir-looking image is combined with an off-nadir image taken from the adjacent orbit with the spacecraft being tilted up to $20^{\circ}$.

For our analysis, two NAC-L images $(0.5 \mathrm{~m} /$ pix image resolution) from subsequent orbits 1943 and 1944 (DOY 322 2009, primary mission) of the Apollo 14 landing site are used. The image pair NAC-L/NAC-L overlaps by $10.3 \%$ under perspective offsets, where stereo processing is possible. The solar incidence angle is $58^{\circ}$ for the image from orbit 1943 and $57^{\circ}$ for the image from orbit 1944 ( $\Delta t \approx 2 \mathrm{~h}$ ). The DTM is computed at a grid size of $2 \mathrm{~m}$, which is about half the LOLA spot size. The processing of the DTM was carried out by an adapted version of the DLR photogrammetric processing system, which was originally implemented for data from the Mars Express High Resolution Stereo Camera (HRSC) (Gwinner et al., 2009, 2010).

\subsection{LROC WAC}

The Wide Angle Camera (WAC) on-board LRO provided nearcomplete coverage of the lunar surface in stereo. The resulting DTM, GLD100 (Scholten et al., 2012), has a grid size of $100 \mathrm{~m}$ and covers the Moon from $-79^{\circ}$ to $+79^{\circ}$ latitude.

\subsection{MESSENGER MDIS and MLA}

The MESSENGER (MErcury Surface, Space ENvironment, GEochemistry and Ranging) spacecraft has completed three Mercury flybys and entered Mercury orbit in March 2011 (Solomon et al., 2011a,b). We have analyzed tracks of the Mercury Laser Altimeter (MLA) from the first Mercury flyby (Zuber et al., 2008) and matched them with a DTM derived from stereo data of the Mercury Dual Imaging System (MDIS) obtained during the second and third flybys of the spacecraft (Preusker et al., 2010). The DTM pixel resolution is $1 \mathrm{~km}$ in contrast to the MLA spot size which varies between 23 and $134 \mathrm{~m}$ during the flyby (Zuber et al., 2008).

\subsection{MGS MOLA and MEX HRSC}

The Mars Global Surveyor (MGS) entered Mars polar orbit in 1997. The on-board Mars Orbiter Laser Altimeter (MOLA) was in operation until June 30, 2001 (Neumann et al., 2002) and has obtained 600 million range measurements (Neumann et al., 2003) with a spot size of $\sim 75 \mathrm{~m}$ (Neumann et al., 2003). Mars Express (MEX) was launched in June 2003 and began its commissioning phase in January 2004 (Chicarro, 2005). The on-board High Resolution Stereo Camera (HRSC) is a nine-look along-track stereo camera system. The DTM used in this work has a resolution of $100 \mathrm{~m}$ per pixel.

\section{Method}

The registration of the two data sets is performed by minimizing the height differences of the $n$ laser shots and the underlying DTM

$$
\sum_{i=0}^{n}\left(h_{d t m_{i}}-h_{\text {laser }_{i}}\right)^{2} \rightarrow \min
$$

with $h_{\text {laser }_{i}}$ being the $i$-th height value measured by the laser and $h_{d t m_{i}}$ the corresponding height in the DTM. A grid search based algorithm shifts the whole track from its nominal position in a user-defined search window, e.g. $l= \pm 50$ pixels in line and $s= \pm 50$ pixels in sample direction and extracts the height value at each position from the DTM using a bilinear interpolation. At each shifted position the standard deviation is determined by

$\sigma_{h_{l, s}}=\sqrt{\frac{\sum_{i=0}^{n}\left(h_{d t m_{i, s}}-h_{\text {laser }_{i}}\right)^{2}}{n-1}}$

The best match between the data sets at pixel size is found at the minimum of Eq. (2). By dividing the pixel position in a userdefined sub-pixel grid, e.g. step size of $1 / 30$ pixel in line and sample direction, and using Eq. (2) again, a sub-pixel position of the best match can be found

$\sigma_{h_{l+s u b_{l}, s+s u b_{s}}}=\sqrt{\frac{\sum_{i=0}^{n}\left(h_{d t m_{i, l+s u b}, s+s u b_{s}}-h_{\text {laser }_{i}}\right)^{2}}{n-1}}$

From the sub-pixel position, translation parameters $x, y, z$ in sample, line, $h$ direction or longitude, latitude, $h$ respectively can 
be extracted. In a second step, we wish to determine the accuracies of these parameters by means of least-squares techniques using the Gauss-Helmert model. The functional model is

$F\left(L, X_{0}\right)=L_{i}-f\left(x+\Delta x_{0}, y+\Delta y_{0}\right)-\left(z+\Delta z_{0}\right)=0$

$L_{i}$ is the observation vector containing the laser heights, $f\left(x+\Delta x_{0}\right.$, $\left.y+\Delta y_{0}\right)$ is a bilinear interpolation of DTM heights at the laser position and $z+\Delta z_{0}$ is the vertical offset between the two data sets. To the translation parameters $(x, y, z)$ that were derived by the grid search approach, we add the initial values $\Delta x_{0}, \Delta y_{0}, \Delta z_{0}$ representing the unknown vector $X_{0}$ in the least-squares approach. The initial values are all set to 0 . The condition is that the difference of each laser height and the interpolated DTM height at the laser spot position corrected for the offset should be 0 .

In order to give accuracies for the resulting fit of the laser track with the DTM, the variance covariance matrix of the adjusted unknowns, $\Sigma_{\hat{x} \hat{x}}$, has to be evaluated:

$\Sigma_{\hat{x} \hat{x}}=s_{0}^{2} Q_{\hat{x} \hat{x}}$

On the main diagonal we find the squares of $\sigma_{\Delta x_{0}}, \sigma_{\Delta y_{0}}, \sigma_{\Delta z_{0}}$ pointing in sample, line and $h$ direction (longitude, latitude, $h$ ) respectively. If the result of the grid search is correct then the resulting $\Delta x_{0}, \Delta y_{0}$, $\Delta z_{0}$ should not be significant, therefore numerically smaller than their corresponding accuracy levels $\sigma_{\Delta x_{0}}, \sigma_{\Delta y_{0}}, \sigma_{\Delta z_{0}}$.

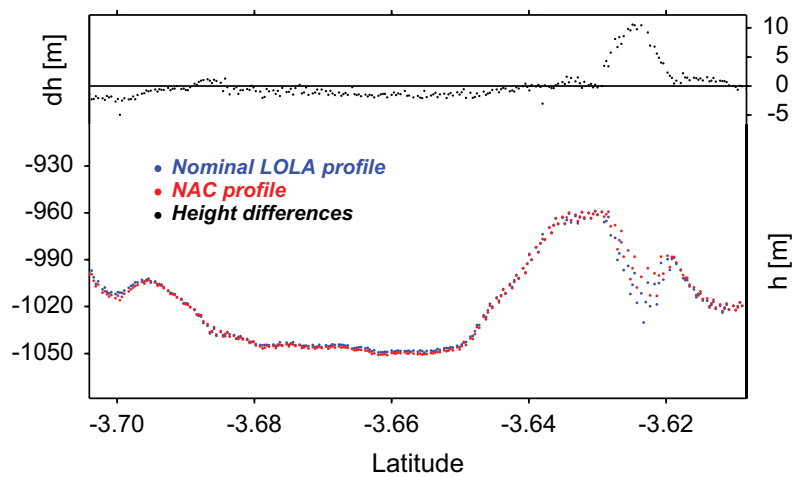

Fig. 1. The nominal LOLA profile and the corresponding DTM heights. At the top of the plot the difference between the two profiles is shown. Height values are given in meters with respect to a sphere with $R=1737.4 \mathrm{~km}$. (For interpretation of the references to color in this figure caption, the reader is referred to the web version of this article.)

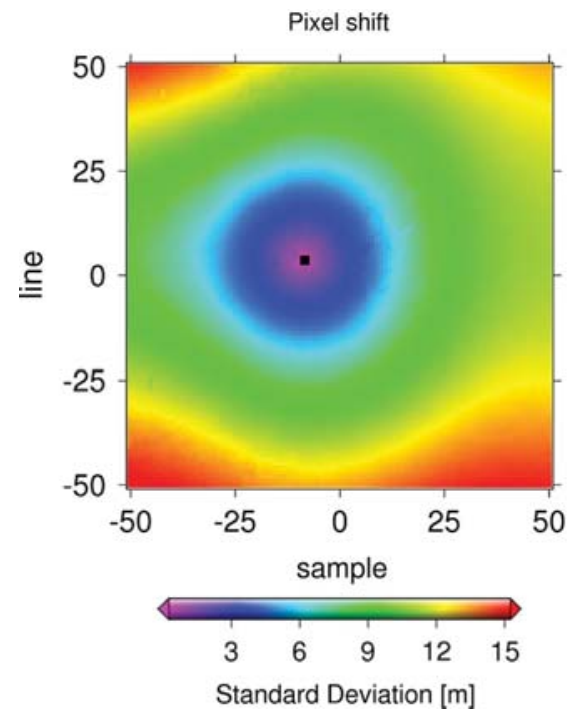

\section{Results}

LRO LOLA-NAC: A sample analysis for co-registering a LOLA track (243 laser spots) with an Apollo 14 landing site DTM is shown in the following. The nominal position of the LOLA track from orbit 3508 and the corresponding DTM heights are plotted in Fig. 1. Significant differences in profile heights are noted (standard deviation of residuals: approximately $2.6 \mathrm{~m}$ ). However, the differences are systematic (probably due to pointing or orbit errors) and can be corrected. Small horizontal offsets of a laser track from its correctly matched position can result in substantial vertical offsets to the reference topographic model when a laser track passes through rough terrain, while vertical differences on flat terrain are comparably small (see Fig. 1). Craters constrain the horizontal and vertical shifts and are ideal for co-registration. The first step of the grid search algorithm returns an integer pixel shift, see Fig. 2, left plot. Around this integer pixel position a sub-pixel grid search with a step size of $1 / 30$ pixel is performed, see Fig. 2 right plot, for refinement. Applying the translations and re-plotting the two profiles, the standard deviation of the height differences drops to $21.22 \mathrm{~cm}$, see Fig. 3. The height differences vary within an interval of $\pm 50 \mathrm{~cm}$, which probably originate mostly from differences in the effective height resolution of the DTM and the laser profile.

Least-squares result: The standard deviations of the adjusted unknowns are $\sigma_{\Delta x_{0}}=8.75 \mathrm{~cm}, \sigma_{\Delta y_{0}}=8.71 \mathrm{~cm}$ and $\sigma_{\Delta z_{0}}=1.60 \mathrm{~cm}$

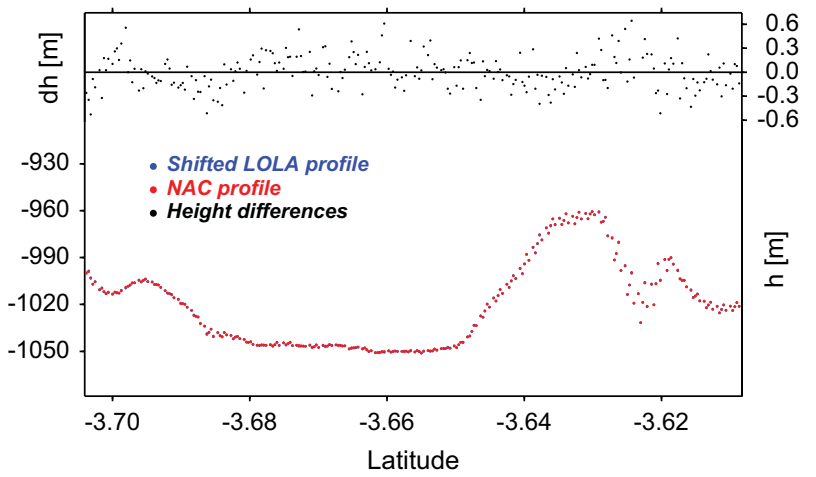

Fig. 3. DTM heights and the corresponding LOLA profile after applying the final translation parameters. The misplacement between the two data sets is minimal. (For interpretation of the references to color in this figure caption, the reader is referred to the web version of this article.)

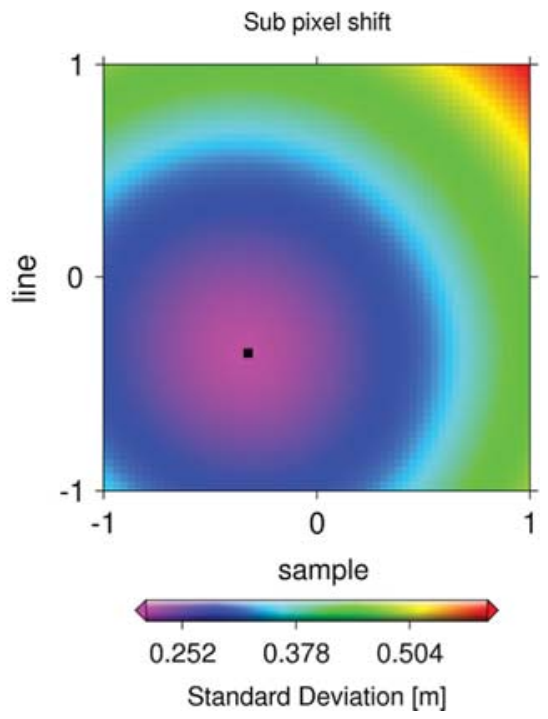

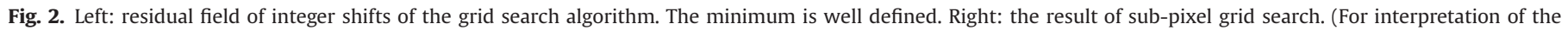
references to color in this figure caption, the reader is referred to the web version of this article.) 
(see Section 4), which attest to the robustness of the fit. The configuration of the NAC DTM with $2 \mathrm{~m}$ resolution and the LOLA laser track with its $5 \mathrm{~m}$ spot size results in a sub-pixel accuracy after the co-registration for both data sets.

LRO LOLA-WAC: The shift needed to co-register the LOLA profile from orbit 10,601 with a subset of the GLD100 is adequate for the entire profile. Even though the track extends over $300 \mathrm{~km}$, there are no obvious rotational offsets, scale differences or trends. The standard deviation of the height differences after the registration is $19.12 \mathrm{~m}$, which is less than 1/5 DTM pixel.

The main problem when matching DTMs with relatively large DTM pixels and laser profiles with high spot to spot densities is that multiple, accurately measured laser height values are compared with similar or even the same interpolated, smoothed DTM pixels. In the example shown in Fig. 4 it can be seen that $\sim 7$ LOLA spots fall within the same DTM pixel and therefore will be compared to almost the same height value retrieved by the interpolation in the DTM, which introduces high correlation in the least-squares adjustment. For this purpose we co-registered 3 versions of LOLA profile 10,601 to the GLD100 resulting in a standard deviation of height residuals of $19-20 \mathrm{~m}$. First we took the original profile with all 25,920 spots, which lead to $\sigma_{\Delta x}=2.47 \mathrm{~m}, \sigma_{\Delta y}=1.54 \mathrm{~m}, \sigma_{\Delta z}=6.11 \mathrm{~m}$. For the same profile three spots have then been averaged resulting in 8642 spots and $\sigma_{\Delta x}=5.33 \mathrm{~m}, \sigma_{\Delta y}=0.71 \mathrm{~m}, \sigma_{\Delta z}=2.89 \mathrm{~m}$. Finally averaging the 5-spot pattern of LOLA to 1 spot and skipping every other

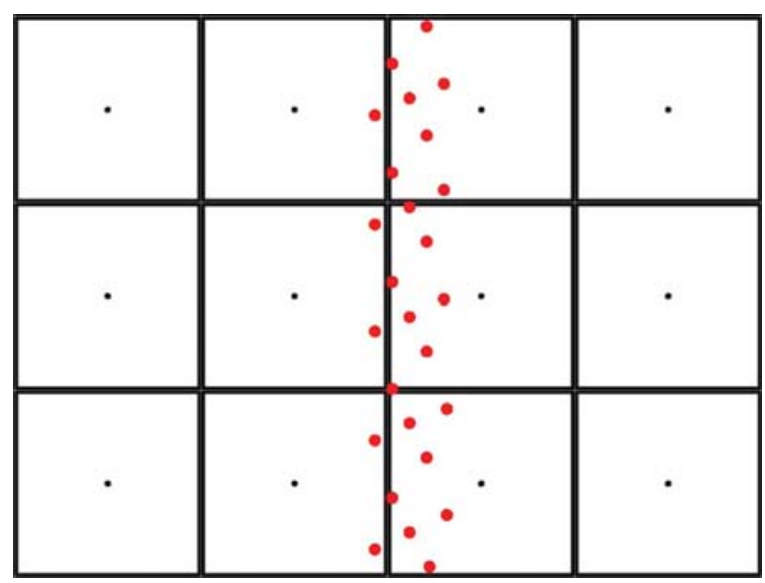

Fig. 4. Distribution of LOLA points on the $100 \mathrm{~m}$-WAC-DTM GLD100. About 7 LOLA spots (red dots) fall into the same DTM pixel (black squares) which introduces high correlations between the height observations of the laser and the interpolated DTM heights. (For interpretation of the references to color in this figure caption, the reader is referred to the web version of this article.)

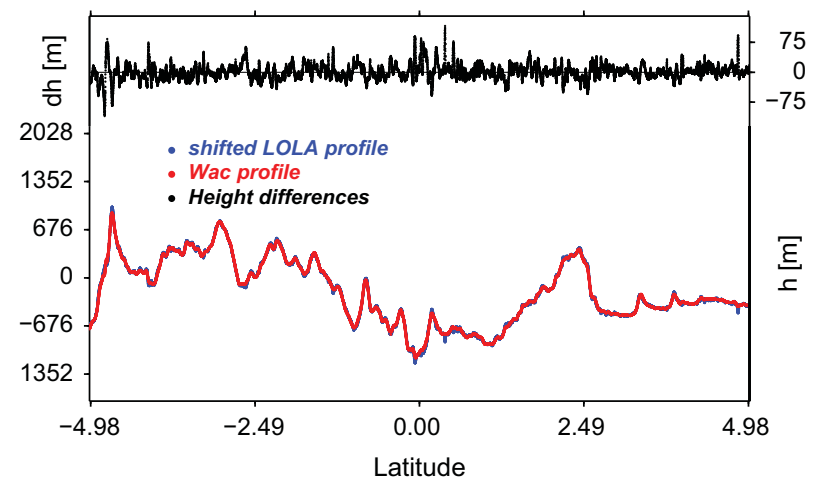

Fig. 5. LOLA track 10,601 on the GLD100. The height differences of the $300 \mathrm{~km}$ long profile, containing 25,920 laser shots show excellent agreement without obvious residual offsets and trends. (For interpretation of the references to color in this figure caption, the reader is referred to the web version of this article.) lead to 2611 spots and $\sigma_{\Delta x}=3.23 \mathrm{~m}, \sigma_{\Delta y}=4.77 \mathrm{~m}, \sigma_{\Delta z}=0.38 \mathrm{~m}$. The horizontal accuracy of the fit is best for the original profile $\left(\sigma_{\Delta x y}=2.91 \mathrm{~m}\right)$ and is degrading the more the profile is averaged $\left(\sigma_{\Delta x y}=5.37 \mathrm{~m}, \sigma_{\Delta x y}=5.76 \mathrm{~m}\right)$. On the other hand the high spot density of the original profile in comparison to a GLD100 pixel leads to inferior vertical accuracy of the fit compared to the averaged profiles, which is described by the correlation in the least-squares adjustment (Fig. 5).

Table 1

Overview of comparisons shown in this work. The first column lists the pixel-sizes of the DTMs $(D)$ used in this work, the second column shows the size of the footprints of the laser altimeters $(L)$. The third column is the ratio of laser spot size and the DTM pixel. The forth and fifth column show the standard deviation of height differences between the two data sets after co-registration and the standard deviation of the fit. In the last two columns we show the ratio with the standard deviation of the fit.

\begin{tabular}{lclllllr}
\hline Missions & $D(\mathrm{~m})$ & $L(\mathrm{~m})$ & $\begin{array}{l}L / D \\
(-)\end{array}$ & $\begin{array}{l}\sigma_{\Delta h} \\
(\mathrm{~m})\end{array}$ & $\begin{array}{l}\sigma_{\text {fit }} \\
(\mathrm{m})\end{array}$ & $\begin{array}{l}L / \sigma_{\text {fit }} \\
(-)\end{array}$ & $\begin{array}{l}D / \sigma_{\text {fit }} \\
(-)\end{array}$ \\
\hline LOLA-NAC & 0.5 & $5^{\mathrm{a}}$ & 10 & 0.21 & 0.16 & 31.25 & 3.12 \\
LOLA-WAC & 100 & $5^{\mathrm{a}}$ & 0.05 & 19.07 & 6.76 & 0.74 & 14.79 \\
MLA-MDIS & 250 & $28-35^{\mathrm{b}}$ & $0.112-0.14$ & 79.18 & 50.37 & $0.55-0.69$ & 4.96 \\
MOLA-HRSC & 100 & $75^{\mathrm{c}}$ & 0.75 & 50 & 13.43 & 5.58 & 7.45 \\
\hline
\end{tabular}

a Smith et al. (2010).

b The distance from the MESSENGER spacecraft to the surface of Mercury for the shown example (see Fig. 7) varied from $440.050 \mathrm{~km}\left(36.005^{\circ} \mathrm{N}\right)$ to $346.979 \mathrm{~km}$ $\left(53.9905^{\circ} \mathrm{N}\right)$. With a beam divergence of $80 \mu \mathrm{rad}$ for MLA, the resulting laser spot sizes therefore vary between $35 \mathrm{~m}$ and $28 \mathrm{~m}$.

${ }^{c}$ Neumann et al. (2003).

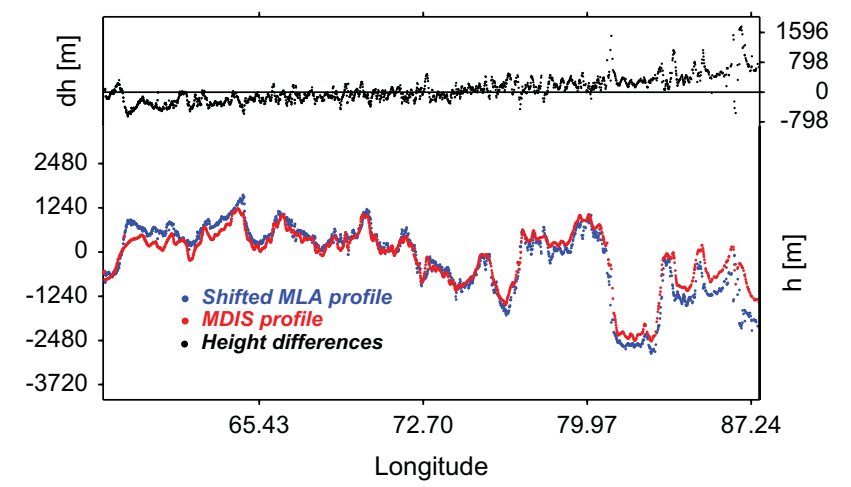

Fig. 6. MLA track matched with an MDIS DTM, computed from stereo images obtained during different flybys. (For interpretation of the references to color in this figure caption, the reader is referred to the web version of this article.)

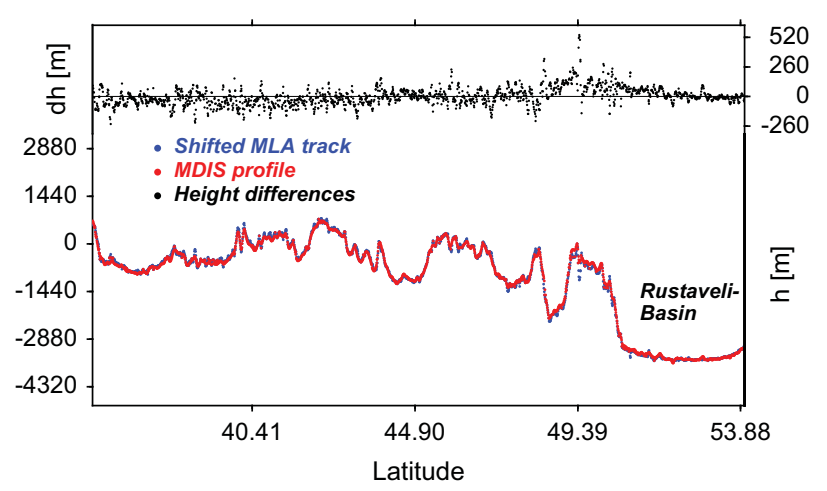

Fig. 7. MLA laser track matched with an MDIS DTM from the MESSENGER orbital mission. The good match of the data sets suggests that altimeter, image, and navigation data are consistent. (For interpretation of the references to color in this figure caption, the reader is referred to the web version of this article.) 


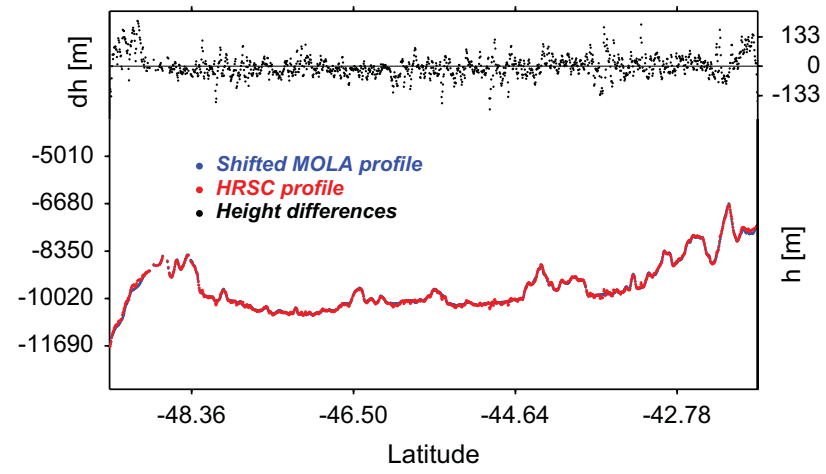

Fig. 8. Shifted MOLA track \#12811 and HRSC DTM. (For interpretation of the references to color in this figure caption, the reader is referred to the web version of this article.)

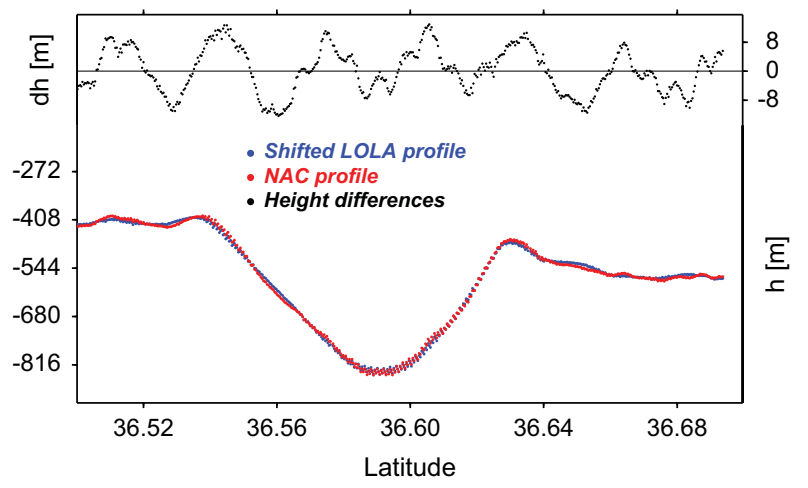

Fig. 9. Shifted LOLA track 1619 and NAC DTM (Orbits 598, 599). Jitter in the DTM is clearly visible in the resulting height differences and a frequency of $\sim 1.7 \mathrm{~Hz}$ can be observed. (For interpretation of the references to color in this figure caption, the reader is referred to the web version of this article.)

\section{Other missions}

For other planetary data sets the relationship between DTM grids and laser profiles is usually quite different than for LRO data regarding laser shot spacing, laser spot sizes, DTM pixel sizes, and data accuracy (see Table 1).

MESSENGER MLA-MDIS: An MLA track from the first Mercury flyby (Zuber et al., 2008) is matched with an MDIS DTM obtained during the second and third flybys of the spacecraft (Preusker et al., 2010) under difficult conditions, such as drastically changing spacecraft range and off-nadir pointing (see Fig. 6). With a laser spot size of $\sim 1 / 10$ of a DTM pixel the geometric relationship between the two data sets is similar to the LOLA-WAC example described earlier. The MLA track from the first flyby consists of 2360 laser points. After matching with the DTM, the standard deviation of the height differences is $311 \mathrm{~m}$ in contrast to $349 \mathrm{~m}$ at the nominal position. A slope of $\sim 0.04^{\circ}$ between the two profiles is identified, suggesting that probably errors existed in the navigation data of the early flybys. More recent DTMs and MLA tracks from the orbital mission phase, processed with more accurate knowledge of orbital position and attitude, lead to smaller residual offsets. The displayed MLA track (see Fig. 7) was recorded in April 2011 and cross-cuts crater Rustaveli. The accuracy of the fit is $\sigma_{\Delta x}=33.88 \mathrm{~m}, \sigma_{\Delta y}=37.19 \mathrm{~m}$ and $\sigma_{\Delta z}=2.43 \mathrm{~m}$ with a standard deviation of height residuals of $79.18 \mathrm{~m}$.

MGS MOLA-MEX HRSC: Another example is given by matching MOLA tracks with HRSC DTMs. Our sample HRSC DTM, covering parts of the area of Argyre Planitia below $40^{\circ}$ south, is very rough and has a high dynamic height range. The example (Fig. 8) shows parts of the shifted MOLA track from orbit 12,811 on the DTM, with which 1387 shots were co-registered. Given a laser spot size of $\sim 75 \mathrm{~m}$ (Neumann et al., 2003) and a DTM pixel size of $100 \mathrm{~m}$, the geometric relationship between DTM grid and

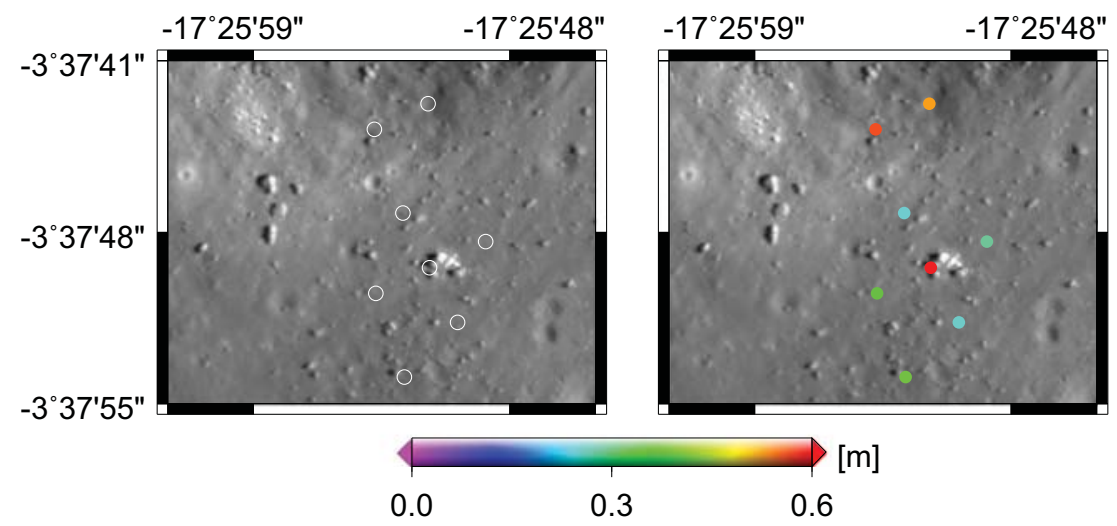

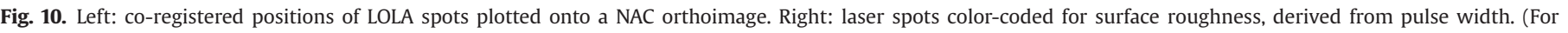
interpretation of the references to color in this figure caption, the reader is referred to the web version of this article.)

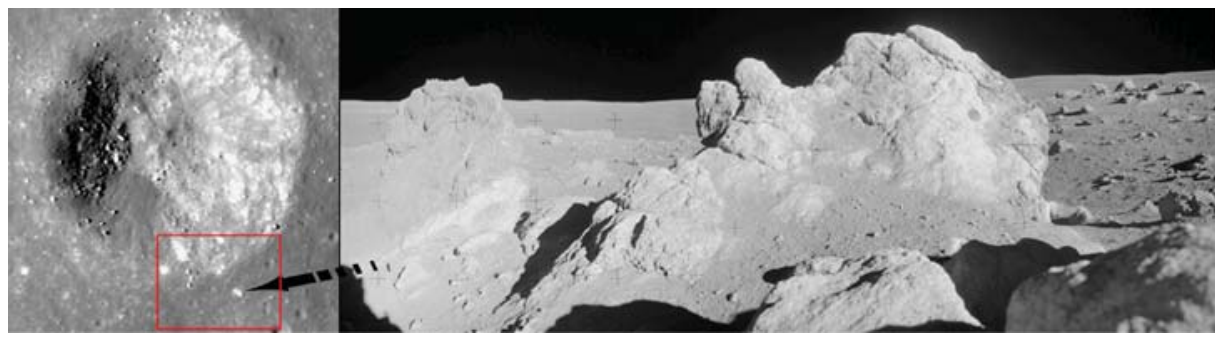

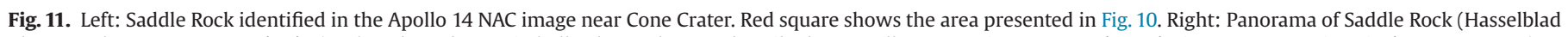

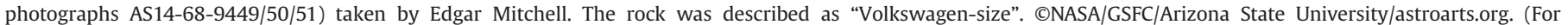
interpretation of the references to color in this figure caption, the reader is referred to the web version of this article.) 
laser spots is different to the examples shown before. The standard deviation of the height differences improved from $65 \mathrm{~m}$ before to $50 \mathrm{~m}$ after matching, which is half the pixel size of the DTM. The accuracy of the fit is $\sigma_{\Delta x_{0}}=9.1 \mathrm{~m}, \sigma_{\Delta y_{0}}=9.78 \mathrm{~m}$ and $\sigma_{\Delta z_{0}}=1.35 \mathrm{~m}$.

Review of the results: In Table 1 we summarize the results of this study. It is comprehensible that if the ratios of the laser footprint and DTM pixel size to the accuracy of the co-registration $\left(L / \sigma_{\text {fit }}\right.$ and $\left.D / \sigma_{\text {fit }}\right)$ shown in the last two columns are $>1$, accurate fits can be made (at sub laser footprint and subpixel level respectively). The number of measurements and the accuracy of the data itself is very crucial but as a rule of thumb we can derive that larger ratios of $L / D$ result in accuracies in the sub pixel level and laser footprint scale. We find that laser spots greater than $75 \%$ (see MOLA-HRSC and LOLA-NAC) of the DTM pixel are favorable for co-registering.

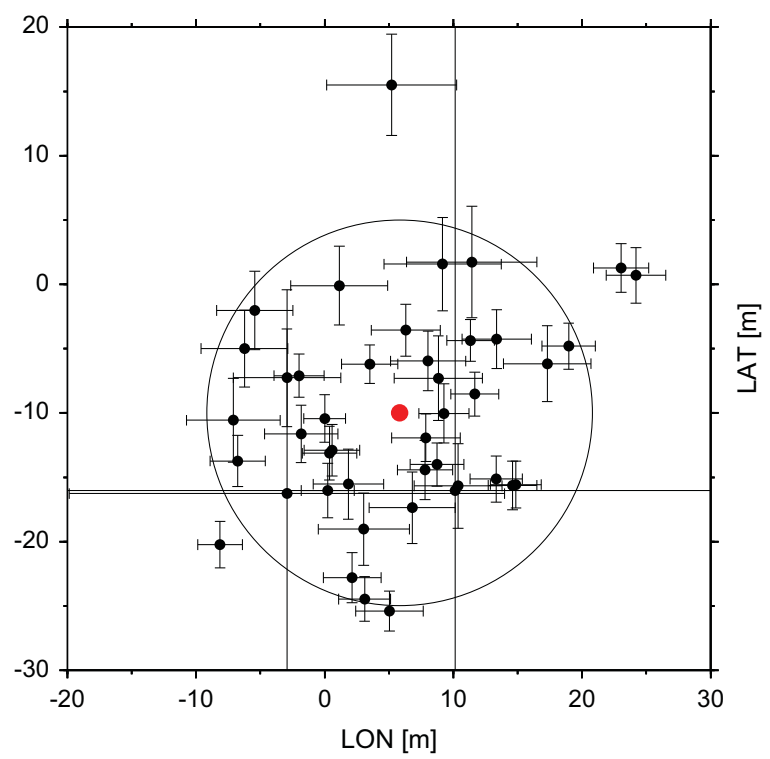

Fig. 12. Offsets of 43 crossover improved LOLA tracks to an Apollo 17 NAC DTM with $1.5 \mathrm{~m}$ pixel resolution. The error bars (magnified by a factor of 10 ) vary from $15 \mathrm{~cm}$ to $4 \mathrm{~m}$ with an average of $\sim 50 \mathrm{~cm}$. The majority of the offsets ( 90\%) scatter around the mean in a circle of $\sim 15 \mathrm{~m}$ radius, which is the total position accuracy of the crossover improved LRO orbits (Mazarico et al., 2012). (For interpretation of the references to color in this figure caption, the reader is referred to the web version of this article.)

\section{Applications and future work}

A correct relative position of the laser track on the DTM is needed for many different applications in planetary mapping and science.

- A comparison of the elevation profiles retrieved from a DTM and a laser profile may be used to assess the quality of the individual data products. Effects like image jitter (see Fig. 9), erroneous DTM trends (see Fig. 6), perhaps due to errors in the relevant navigation data, can be detected and corrected for.

- After co-registration with DTMs, direct comparisons can be made between LOLA and orthoimages (which are typically derived from the same DTMs) at the limits of data resolution. In particular, with the correct laser spot position, roughness derived from the laser pulse width (Neumann et al., 2003; Smith et al., 2001; Garvin et al., 1999) can be compared with the orthoimage, and a calibration of laser pulse width for correct surface roughness can be made. The derived roughness can also be compared to the actual surface morphology in the image in full detail (see Fig. 10). Note the correlation of color coded laser pulse spread with surface morphology, the two red data points ( $5 \mathrm{~m}$ diameter) indicate high roughness as seen by the laser. The lower red colored laser point marks a rock; most likely this is 'Saddle Rock' visited by the Apollo 14 astronauts (Alan Shepard and Edgar Mitchell) (see Fig. 11).

- Offsets between a camera derived DTM and laser tracks of the same orbiter can be indicative for misalignment between the two instruments. We detect persistent offsets between LOLA tracks and the NAC DTM, which are a strong indicator for small residual errors in the nominal pointing kernels (see Fig. 12).

- A comparison of the topographic profiles of a DTM and a laser can be used to assess the effective height resolution of the DTM. While the DTM grid size alone does not necessarily represent its own effective resolution, a comparison of the DTM with the laser track, considered highly accurate, is helpful. By introducing a priori standard deviations for the laser and DTM heights, statistical tests $\left(\chi^{2}\right)$ can be used to test if chosen parameters are appropriate. Hence, a statistically verified standard deviation of DTM heights can be given (Oberst et al., 2010a).

- Co-registration offsets may be used to identify map-tie problems, i.e. inconsistencies in the coordinate system parameters or erroneous assumptions in planet rotational model. A first analysis of MDIS DTMs suggests that comparisons with multiple laser tracks taken at different times can provide an observation for the librational motion of Mercury. Periodic shifts and offsets
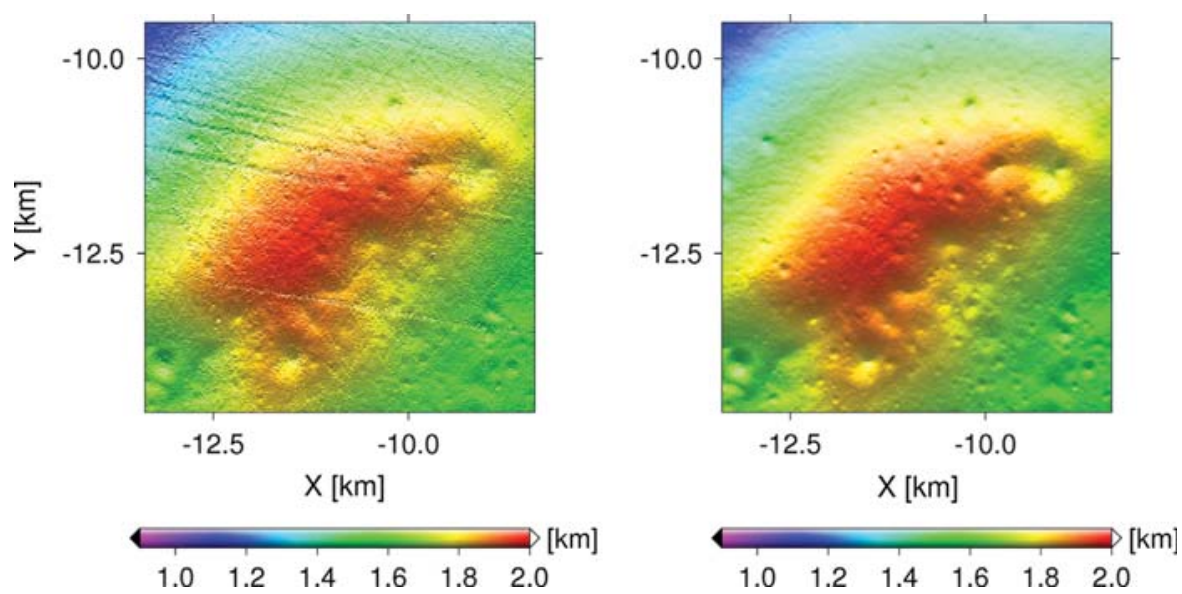

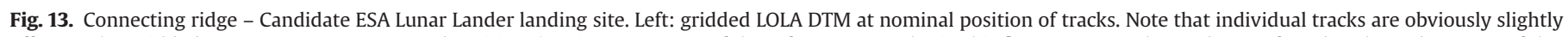
offset. Right: gridded LOLA DTM at co-registered position. (For interpretation of the references to color in this figure caption, the reader is referred to the web version of this article.) 
have been detected by the introduced algorithm (Stark et al. 2012).

- Correctly co-registered laser tracks may be introduced to help improve terrain models, in particular to redo the bundle adjustment of the images. The registered laser track spots will serve as control points at fixed lateral and vertical coordinates. Likewise, misplaced laser tracks can be identified, corrected, or removed to yield improved gridded laser terrain models. Such work is currently in progress within our team to generate lunar landing site DTMs for the European Space Agency (ESA) (see Fig. 13; Carpenter et al., 2012; De Rosa et al., 2012).

\section{Summary}

It could be shown that the algorithm finds a best fit with subpixel accuracy between laser profiles and DTMs. Further a reasonable amount of laser shots is required to have a statistically reliable result. The use of this routine is not limited to one specific data set like LRO but is kept fairly independent of required data formats and missions. The range of application is big and the list we provided is most certainly incomplete. An overview of DTM pixel sizes, laser spot sizes, and the resulting standard deviations of height differences after matching is given in Table 1.

\section{Acknowledgments}

P. Gläser was funded by a Grant of the German Research Foundation (FOR 1503, OB 124/8-1) and I. Haase was funded by a Grant of the German Space Agency (FKZ 50OW1202). We wish to thank the LOLA and LROC Science Team for releasing such great data products. We wish to thank Frank Scholten and Frank Preusker (both DLR) who provided stereo DTMs used in this study including helpful discussions. Furthermore, we wish to thank our colleagues Sven Weisbrich, Christian Manthe, Steffi Burmeister, Konrad Willner (all TU Berlin) and Alexander Stark (DLR) for many fruitful discussions.

\section{References}

Carpenter, J.D., Fisackerly, R., De Rosa, D., Hondou, B., 2012. Scientific preparations for lunar exploration with the European Lunar Lander. Planetary and Space Science 74, 208-223 1207.4965.

Chicarro, A.F., 2005. The Mars express mission - summary of scientific results from orbit. In: Mackwell, S., Stansbery, E. (Eds.), 36th Annual Lunar and Planetary Science Conference, p. 1035.

De Rosa, D., Bussey, B., Cahill, J.T., Lutz, T., Crawford, I.A., Hackwill, T., van Gasselt, S. Neukum, G., Witte, L., McGovern, A., Grindrod, P.M., Carpenter, J.D., 2012 Characterisation of potential landing sites for the European Space Agency's Lunar Lander project. Planetary and Space Science 74, 224-246 1208.5587.

Di, K., Hu, W., Liu, Y., Peng, M., 2012. Co-registration of Chang'E-1 stereo images and laser altimeter data with crossover adjustment and image sensor mode refinement. Advances in Space Research 50, 1615-1628.

Garvin, J.B., Frawley, J.J., Abshire, J.B., 1999. Vertical roughness of Mars from the Mars Orbiter Laser Altimeter. Geophysical Research Letters 26, 381-384.

Gläser, P., Haase, I., Scholten, F., Oberst, J., 2010. Precision registration of LRO altimeter tracks and stereo terrain models - implications for surface slopes and roughness. In: European Planetary Science Congress 2010, p. 296

Gwinner, K., Scholten, F., Preusker, F., Elgner, S., Roatsch, T., Spiegel, M., Schmidt, R. Oberst, J., Jaumann, R., Heipke, C., 2010. Topography of Mars from global mapping by HRSC high-resolution digital terrain models and orthoimages: characteristics and performance. Earth and Planetary Science Letters 294, 506-519.

Gwinner, K., Scholten, F., Spiegel, M., Schmidt, R., Giese, B., Oberst, J., Heipke, C., Jaumann, R., Neukum, G., 2009. Derivation and validation of high-resolution digital terrain models from Mars express HRSC data. In: Photogrammetric Engineering and Remote Sensing, pp. 1127-1142.
Haase, I., Oberst, J., Scholten, F., Wählisch, M., Gläser, P., Karachevtseva, I., Robinson, M.S. 2012. Mapping the Apollo 17 landing site area based on Lunar Reconnaissance Orbiter camera images and apollo surface photography. Journal of Geophysical Research (Planets) 117, 0.

Kim, J., Muller, J.P., Morley, J., 2000. Automated MOLA track registration in MOC and VIKING images and it's application for the establishment of new 3-D control points on Mars, vol. 33.

Kolb, K.J., Okubo, C.H., 2009. Coregistration of Mars orbiter laser altimeter (MOLA) topography with high-resolution mars images. Computers and Geosciences 35, 2304-2313.

Lin, S.Y., Muller, J.P., Mills, J.P., Miller, P.E., 2010. An assessment of surface matching for the automated co-registration of MOLA, HRSC and HiRISE DTMs. Earth and Planetary Science Letters 294, 520-533.

Mazarico, E., Rowlands, D.D., Neumann, G.A., Smith, D.E., Torrence, M.H., Lemoine, F.G., Zuber, M.T., 2012. Orbit determination of the Lunar Reconnaissance Orbiter. Journal of Geodesy 86, 193-207.

Neumann, G.A., Abshire, J.B., Aharonson, O., Garvin, J.B., Sun, X., Zuber, M.T., 2003. Mars orbiter laser altimeter pulse width measurements and footprint-scale roughness. Geophysical Research Letters 30 110000-1.

Neumann, G.A., Abshire, J.B., Smith, D.E., Sun, X., Zuber, M.T., 2002. MOLA 1064 nm radiometry measurements: status and prospects in extended mission. In: Lunar and Planetary Institute Science Conference Abstracts, p. 1889

Oberst, J., Preusker, F., Phillips, R.J., Watters, T.R., Head, J.W., Zuber, M.T., Solomon, S.C., 2010a. The morphology of Mercury's Caloris basin as seen in MESSENGER stereo topographic models. Icarus 209, 230-238.

Oberst, J., Scholten, F., Matz, K.D., Roatsch, T., Wählisch, M., Haase, I., Gläser, P., Gwinner, K., Robinson, M.S., Lroc Team, 2010b. Apollo 17 landing site topography from LROC NAC stereo data - first analysis and results. In: Lunar and Planetary Institute Science Conference Abstracts, pp. 2051-+.

Preusker, F., Oberst, J., Blewett, D.T., Gwinner, K., Head, J.W., Murchie, S.L., Robinson, M. S., Watters, T.R., Zuber, M.T., Solomon, S.C., 2012. Topography of Mercury from stereo images: first samples from MESSENGER orbital mapping. In: Lunar and Planetary Institute Science Conference Abstracts, p. 1913.

Preusker, F., Oberst, J., Phillips, J., Watters, T.R., Head, J.W., Zuber, M.T., Turner, F.S. Solomon, S.C., 2010. Digital terrain models of Mercury from MESSENGER stereo images. In: Lunar and Planetary Institute Science Conference Abstracts, p. 1789.

Scholten, F., Oberst, J., Matz, K.D., Roatsch, T., Wählisch, M., Speyerer, E.J., Robinson, M.S. 2012. GLD100: the near-global lunar $100 \mathrm{~m}$ raster DTM from LROC WAC stereo image data. Journal of Geophysical Research (Planets) 117, 0.

Smith, D.E., Zuber, M.T., 2000. An overview of the topography of Mars from the Mars Orbiter Laser Altimeter (MOLA). In: Second International Conference on Mars Polar Science and Exploration, p. 159.

Smith, D.E., Zuber, M.T., Albee, A.L., 2000. The shape and topography of Mars from the Mars Orbiter Laser Altimeter (MOLA). In: IAU Joint Discussion.

Smith, D.E., Zuber, M.T., Frey, H.V., Garvin, J.B., Head, J.W., Muhleman, D.O., Pettengill, G.H., Phillips, R.J., Solomon, S.C., Zwally, H.J., Banerdt, W.B., Duxbury, T.C., Golombek, M.P., Lemoine, F.G., Neumann, G.A., Rowlands, D.D., Aharonson, O. Ford, P.G., Ivanov, A.B., Johnson, C.L., McGovern, P.J., Abshire, J.B., Afzal, R.S., Sun, X., 2001. Mars Orbiter Laser Altimeter: experiment summary after the first year of global mapping of Mars. Journal of Geophysical Research 106, 23689-23722.

Smith, D.E., Zuber, M.T., Jackson, G.B., Cavanaugh, J.F., Neumann, G.A., Riris, H. Sun, X., Zellar, R.S., Coltharp, C., Connelly, J., Katz, R.B., Kleyner, I., Liiva, P., Matuszeski, A., Mazarico, E.M., McGarry, J.F., Novo-Gradac, A.M., Ott, M.N. Peters, C., Ramos-Izquierdo, L.A., Ramsey, L., Rowlands, D.D., Schmidt, S., Scott, V.S., Shaw, G.B., Smith, J.C., Swinski, J.P., Torrence, M.H., Unger, G., Yu, A.W. Zagwodzki, T.W., 2010. The Lunar orbiter laser altimeter investigation on the lunar reconnaissance orbiter mission. Space Science Reviews 150, 209-241.

Smith, D.E., Zuber, M.T., Neumann, G.A., Mazarico, E., Head, J., Torrence, M.H., Lola Science Team, 2011. Results from the Lunar Orbiter Laser Altimeter (LOLA): global, high resolution topographic mapping of the Moon. In: Lunar and Planetary Institute Science Conference Abstracts, p. 2350.

Solomon, S.C., McNutt, R.L., Bedini, P.D., Anderson, B.J., Blewett, D.T., Evans, L.G. Gold, R.E., Krimigis, S.M., Murchie, S.L., Nittler, L.R., Phillips, R.J., Prockter, L.M., Slavin, J.A., Zuber, M.T., 2011a. MESSENGER at Mercury: flyby accomplishments and orbital observing plans. In: Lunar and Planetary Institute Science Conference Abstracts, p. 1781.

Solomon, S.C., McNutt, Jr., R.L., Anderson, B.J., Blewett, D.T., Evans, L.G., Gold, R.E., Krimigis, S.M., Murchie, S.L., Nittler, L.R., Phillips, R.J., Prockter, L.M., Slavin, J.A., Zuber, M.T., 2011b. Mercury after six months of MESSENGER orbital observations. In: EPSC-DPS Joint Meeting 2011, p. 430.

Stark, A., Oberst, J., Preusker, F., Gwinner, K., Peale, S.J., Margot, J.L., Zuber, M.T. Solomon, S.C., 2012. In-situ measurement of Mercury's physical librations using image and laser altimeter data from MESSENGER: general approach and sensitivity analysis. In: Lunar and Planetary Institute Science Conference Abstracts, p. 1389

Yoon, J.S., Shan, J., 2005. Combined adjustment of MOC stereo imagery and MOLA altimetry data, photogrammetric engineering and remote. Sensing 20, 1179-1186.

Zuber, M.T., Smith, D.E., Solomon, S.C., Phillips, R.J., Peale, S.J., Head, J.W., Hauck, S.A., McNutt, R.L., Oberst, J., Neumann, G.A., Lemoine, F.G., Sun, X., Barnouin-Jha, O. Harmon, J.K., 2008. Laser altimeter observations from MESSENGER's first mercury flyby. Science $321,77-79$. 